\title{
A ONE-SENTENCE LINE-OF-SIGHT PROOF OF THE EXTREME VALUE THEOREM
}

\author{
SAMUEL J. FERGUSON
}

\begin{abstract}
We give a one-sentence proof that a continuous real-valued function $\mathrm{f}$ on a closed, bounded interval attains a maximum value, by the following device. We define $\mathrm{x}$ in $[\mathrm{a}, \mathrm{b}]$ to be a lookout point if $f(t)$ does not exceed $f(x)$ whenever $t$ lies in $[a, x)$. Letting $c$ be the maximum of the set of lookout points, we prove that $f(c)$ is the maximum value of $f$.
\end{abstract}

The maximum value of a continuous real-valued function $f$ on $[a, b]$ is attained at its largest "lookout point." We call $x$ in $[a, b]$ a lookout point if, whenever $t$ lies in $[a, x)$, we have

$$
f(t) \leq f(x) \text {. }
$$

The set $L$ of lookout points is closed. Indeed, let $x_{n} \rightarrow x$, with $x_{n}$ in $L$. If $t$ is in $[a, x)$, then eventually $t$ lies in $\left[a, x_{n}\right)$, so

$$
f(t) \leq f\left(x_{n}\right) .
$$

By continuity, $f(t) \leq f(x)$, as desired. We use the fact that a closed, bounded, and nonempty set has a maximum and a minimum. In particular, $\max (L)$ exists.

Extreme Value Theorem. If $f$ is a real-valued continuous function on $[a, b]$ then $f$ has a maximum value on $[a, b]$. In other words, for some $c$ in $[a, b]$, no value of $f$ exceeds $f(c)$.

Proof. Letting

$$
L=\{x \text { in }[a, b] \text { such that } t \text { in }[a, x) \text { implies } f(t) \leq f(x)\}
$$

and $c=\max (L)$, it suffices to show that, given $k>f(c)$, the closed, bounded set

$$
S_{k}=\{t \text { in }[a, b] \text { such that } f(t) \geq k\}
$$

is empty, which follows since, if some $d$ satisfies $f(d) \geq k$, then $d>c$, whence $d$ is not in $L$, so there exists a $t<d$ for which

$$
f(t)>f(d) \geq k,
$$

proving that $S_{k}$ has no minimum.

Courant Institute of Mathematical Sciences, New York University, 251 Mercer St, New YoRK, NY 10012

E-mail address: ferguson@cims.nyu.edu 